\title{
RELIABILITY OF WIRELESS INSOLE BAROPODOMETRY OF NORMAL INDIVIDUAL'S GAIT
}

\section{CONFIABILIDADE DA BAROPODOMETRIA POR PALMILHA SEM FIO NA MARCHA DE INDIVÍDUOS NORMAIS}

\author{
Larissa Barbosa Oliveira ${ }^{1,2}$ (i), Daniel augusto Maranho ${ }^{1,3}$ (1), alberto Cliquet Júnior ${ }^{1}$ (1), \\ mauro Cesar mattos e Dinato ${ }^{1,2}$ (1), Rodrigo Gonçalves Pagnano ${ }^{1}$ (1)
}

1. Universidade Estadual de Campinas, Faculty of Medical Sciences, Department of Orthopedics and Traumatology, Campinas, SP, Brazil.

2. Instituto Vita, São Paulo, SP, Brazil.

3. Hospital Sírio-Libanês, Brasília, DF, Brazil.

\section{ABSTRACT}

Objective: The plantar pressure distribution can be assessed quantitatively by computerized baropodometry such as carpet or insole. An insole-type system with wireless transmission was developed and plantar pressure results were previously validated by force platform. However, the reproducibility of the system had not been determined. Our objective was to evaluate the reliability of the results in different gait cycles, clinical characteristics and in different plantar anatomical sites. Methods: 41 healthy adults (age, $34 \pm 13$ years; body mass index, $25 \pm 5 \mathrm{~kg} / \mathrm{m}^{2} ; 26$ [63\%], male, 26 [63\%] practicing physical activity) were evaluated. Baropodometer evaluations were performed in 3 walking cycles with $100 \mathrm{~m}$ each, and the reliability between the cycles was examined. Pressure points on the heel, first metatarsal, fifth metatarsal and total plantar pressure were analyzed and compared. Results: Moderate agreement was identified between the second and third cycles (ICC, 0.66; 95\% Cl, 0.14-0.83). Physical activity practitioners showed higher total plantar pressure $(70.8$ vs $68.2 \mathrm{Kpa} ; \mathrm{p}=0.04)$ and higher pressure in the heel (70.7 vs $68.1 \mathrm{Kpa} ; \mathrm{p}=0.036$ ) in relation to sedentary ones. Conclusion: The insole was able to assess plant pressure with moderate reliability from the adaptation period. Level of Evidence III, Case control study - Investigating a diagnostic test.

Keywords: Transducers, Pressure. Foot. Monitoring. Gait.

\section{RESUMO}

Objetivo: A distribuição da pressão plantar pode ser avaliada quantitativamente por baropodometria computadorizada tipo tapete ou palmilha. Um sistema tipo palmilha com transmissão sem fio foi desenvolvido, cujos resultados de pressão plantar foram previamente validados por plataforma de força. No entanto, a reprodutibilidade do sistema não havia sido determinada. Nosso objetivo foi avaliar a confiabilidade dos resultados em relação a diferentes ciclos de marcha, características clínicas e em diferentes sítios anatômicos plantares. Métodos: Foram avaliados 41 adultos saudáveis (idade, $34 \pm 13$ anos; índice de massa corpórea, $25 \pm 5$ kg/m2; 26 [63\%], sexo masculino, 26 [63\%] praticantes de atividade física). Avaliações com o baropodômetro foram realizadas em 3 ciclos de marcha com distância de 100 m, e avaliada a concordância entre os ciclos. Pontos de pressão no calcanhar, primeiro metatarsal, quinto metatarsal e a pressão plantar total foram analisados e comparados. Resultados: Houve moderada concordância entre o segundo e terceiro ciclos (CCI, 0,66; IC95\%, 0,14-0,83). Praticantes de atividades físicas apresentaram pressão plantar total (70,8 vs 68,2 Kpa; $p=0,04)$ e no calcanhar (70,7 vs 68,1 Kpa; $p=0,036)$ aumentada em relação aos sedentários. Conclusão: A palmilha foi capaz de avaliar a pressão plantar com confiabilidade moderada a partir do período de adaptação. Nível de Evidência III, Estudo diagnóstico - Investigando um teste diagnóstico.

Descritores: Transdutores de Pressão. Pé. Monitoramento. Marcha.

Citation: Oliveira LB, Maranho DA, Cliquet A Jr, Dinato MCM, Pagnano RG. Reliability of wireless insole baropodometry of normal individual's gait. Acta Ortop Bras. [online]. 2021;29(5):238-241. Available from URL: http://www.scielo.br/aob.

\section{INTRODUCTION}

The quantification and anatomical monitoring of plantar pressure are important in the practice of the foot and ankle surgeon, as the results can help in the decisions of clinical, surgical and rehabilitation treatments. ${ }^{1}$ The evolutionary control of areas of plantar overload allows evaluating individuals over time, and assisting in the preparation of insoles, orthosis and in the indication of surgeries. ${ }^{2}$
Additionally, the development of an objective database on plantar pressure and pressure increasing sites can contribute to scientific research in order to observe the best clinical outcome in sports medicine and foot and ankle surgery. ${ }^{1,3}$

Baropodometry quantitatively evaluates plantar pressure and identifies anatomical landmark of overload. ${ }^{4}$ The current gold standard in plantar pressure assessment is static and dynamic baropodometry in a

All authors declare no potential conflict of interest related to this article.

The study was conducted at Departamento de Ortopedia e Traumatologia da Faculdade de Ciências Médicas da Universidade Estadual de Campinas. Correspondence: Larissa Barbosa de Oliveira. Rua Mato Grosso, 306, 1 floor, São Paulo, SP, Brazil, 01239040. laribaroli@gmail.com 
treadmill or platform device. ${ }^{5}$ However, the treadmill baropodometer has a relatively high cost and evaluates a single step at a time and may not evaluate the gait performed in the usual way. The mobile baropodometers are coupled in insoles and can be used inside the usual shoes or directly applied to the sole. With advances in sensor technology, portability and versatility, it was allowed that insole baropodometers could be used during gait in a common dynamic way, and during sports in different types of environment. ${ }^{6,7}$ With insoles was possible to evaluate plantar pressure under various clinical and research conditions. Nevertheless, validation, reliability or applicability vary between different systems and studies on insole baropodometers. ${ }^{2,3,6-8}$ In our field, Varoto et al. developed a wireless plantar pressure monitoring system, with low-cost data acquisition module, coupled with flexible high-resolution sensor. ${ }^{3}$ The system was validated with a force platform and a pilot study conducted in a single individual based on static and dynamic activities in the biomechanical platform. ${ }^{3}$ However, the reliability of this system in normal individuals had not been studied. The aim of this study was to evaluate the reliability of the results in relation to different gait cycles, clinical variables of volunteers and in different plantar anatomical sites.

\section{MATERIALS AND METHODS}

This study was approved by the Research Ethics Committee of the institution (CAAE: 84979417.9.0000.5404). In total, 45 individuals evaluated plantar pressure with the baropodometric insole system. The individuals had no musculoskeletal complaints in the last six months, nor known deformities or diseases in the foot and ankle, lower limbs, hips and spine. Age ranged from 16 to 65 years and the Brazilian numbering of shoes between 37 and 42 . Four individuals were excluded due to erratic data acquisition by the system, resulting in the final sample of 41 participants. The mean age ( \pm standard deviation) was $34 \pm 13$ years, and 26 (63\%) were male. The mean height was $1.73 \pm 0.09 \mathrm{~m}$; weight, $76 \pm 16 \mathrm{~kg}$ and body mass index (BMI), $25 \pm 5 \mathrm{~kg} / \mathrm{m}^{2}$. The median foot size was shoes number 40 . The right side was dominant in $88 \%$ of individuals, and 26 (63\%) practiced physical activity regularly.

To evaluate plantar pressure, a baropodometer of the insole type was performed. The system consists of a low-cost wireless data acquisition module (less than \$20.00), flexible high-resolution pressure sensor (Medical Sensor 3000, Tekscan, Inc., Boston, MA, USA) and Java application for visualization. ${ }^{3}$ The flexible tactile sensor for measuring pressure distribution consists of two flexible substrates joined by adhesive and dielectric layers. Electrodes of both substrates establish matrix of rows and columns, and each intersection forms detection element that alters electrical resistance when force was applied. ${ }^{3}$ The microcontroller associated with the switching circuit selected the detection element, scanning and performing the conversion from analog into digital of voltage amplifier circuits. The switching circuit, including modified voltage dividers, converted electrical resistances into voltage. The generated data was stored in the module (device coupled to the volunteer's leg), and a microcontroller provided serial data via Bluetooth ${ }^{\circledR}$ for Java application software (Figure 1). ${ }^{3}$

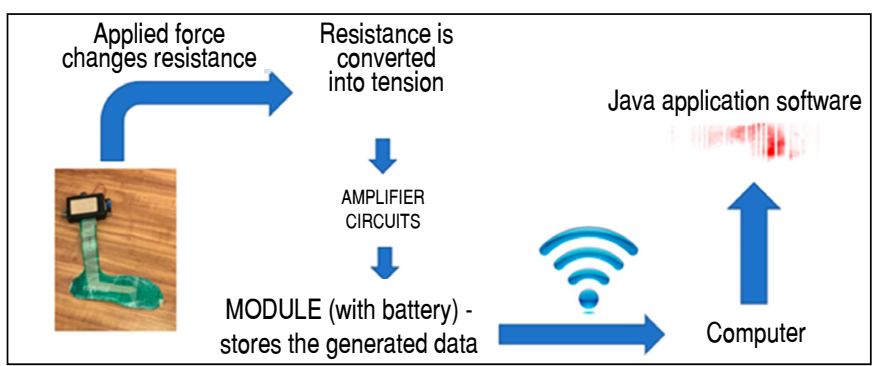

Figure 1. Insole baropometry system demonstration flowchart, developed with wireless transmissibility and low cost components.
For all individuals, the insole was inserted and embedded in the usual sports shoe, in order to avoid interference in gait related to the adaptation of unusual footwear, persisting only individual adaptations secondary to the contact of the foot with the insole. Then, the data acquisition module was fixed to the individual's leg by an elastic band, to make comfortable when walking with the device. A single insole was used by all individuals in all collections. The insole is $26 \mathrm{~cm}$ long, choosing to evaluate the feet whose length varied by up to 2 $\mathrm{cm}$ (24 to $28 \mathrm{~cm}$, which corresponds, on average, to shoe numbers 37 to 42 ), so that the insole fits well to the footwear. The insole was calibrated before the beginning of the collections. With the device properly attached to the right foot, the volunteer was asked to climb on the treadmill (Riguetto, model R-2500Ee, Campinas, SP, Brazil) and the acceleration to start the gait was triggered. The first data collection cycle was started at the time the treadmill reached the pre-established speed of $4 \mathrm{~km} / \mathrm{h}$, and ceased after the volunteer walked $100 \mathrm{~m}$. Then, two other cycles were collected in the right foot, totaling a distance of $300 \mathrm{~m}$. As the insole is flexible and sensors are present on both sides, the same protocol was performed for the left foot, removing the device after the end of the evaluations.

After data collection and transfer via Bluetooth to the computer, the software created an image in two-dimensional format of the insole, characterized by several points with variable color gradient, corresponding to the pixels generated by the pressure applied at each of the points in the sensor (Figure 2A). Four anatomical regions were visually chosen: head of the first and fifth metatarsal, heel and total plantar surface (all pressure points generated in the extension of the plantar surface at that time). The moments of total contact of the foot with the ground were selected. The pixels responsible for generating the colorimetric gradation of the pressures were obtained by converting the pressure values $(\mathrm{kPa})$ according to the mathematical function $y=-0.9889 x+274.47$. For each volunteer, 242 images were generated per cycle, totaling 726 images for each point (1st MTT, 5th MTT, heel and the whole foot - Figure 2B) in each foot. In a total of 3 cycles, 5.808 points were generated for analysis for each of the volunteers. Each data collection took, on average, 20 minutes.

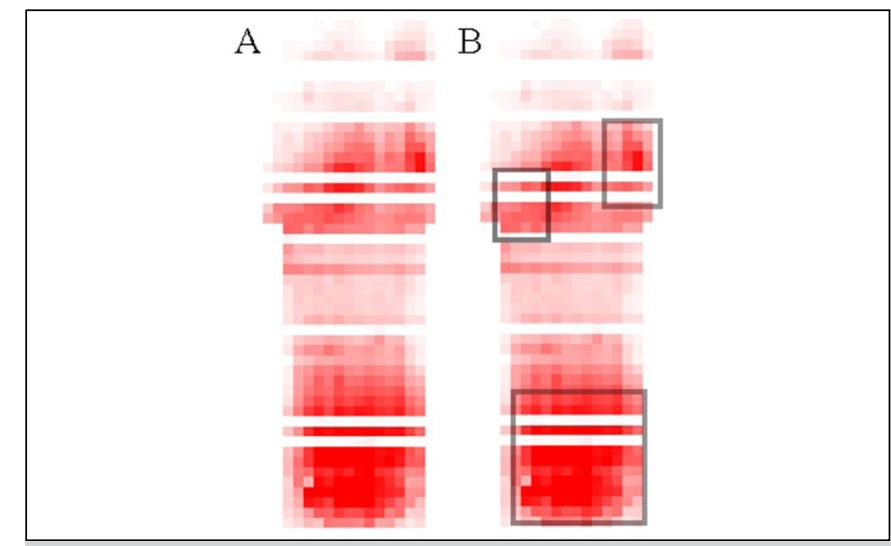

Figure 2. A: Image generated with the foot in medium support; B: Demonstration of selection of the point to be analyzed by the software (in the example we have the selection of the regions of the head of the first and fifth metatarsals and heel).

\section{Statistical Analysis}

Demographic data were presented as frequency and percentage, mean and standard deviation, or median. For each pressure point studied, the means of the pressures of the given pathway were obtained, compared to the demographic variables (sex and physical activity) and the analyzed side. The reliability of the instrument was verified by the Intraclass Correlation Coefficient (ICC) ${ }^{9}$ between 3 
distinct gait sequences from the collections of each individual. The ICC was calculated for each feet, separating the images corresponding to each of the cycles. Thus, we compared the 242 images generated by the pressures of the first $100 \mathrm{~m}$ with the 242 images generated by pressures between 101 and $200 \mathrm{~m}$ and between 201-300 m. ICC values lower than 0.5 indicate low reproducibility; values between 0.5 and 0.75 indicate moderate reproducibility; values between 0.75 and 0.9 indicate good reproducibility and values greater than 0.9 indicate excellent reproducibility. ${ }^{9,10}$ Since it was estimated that the first collection was that of adaptation to the insole, there was potential impairment in relation to reliability when performing the first cycle. Thus, it was decided to perform the statistical analysis of the data of the last collection, that is, between 200-300 m (images 485 to 726). Data normality was verified conducting the Shapiro-Wilk test. Student's t-test or Mann-Whitney test were performed to compare the pressure values between binary variables, and Pearson's coefficient was conducted to evaluate correlation between pressure and $\mathrm{BMI}$ values. Statistical analysis was performed with the software Statistical Package for the Social Sciences (IBM SPSS Statistics, version 24, IBM Corp., Armonk, N.Y., USA) and $p<0.05$ values were considered significant.

\section{RESULTS}

After the second cycle, the agreement between cycles in baropodometry was moderate. Within the first cycle, the ICC was 0.45 (95\% Cl, 0.33 to 0.55$)$, corresponding to low reliability. Between the first and second collections (100-200 m; 243-484 images), the ICC was 0.47 (95\% Cl, 0.34 to 0.57), corresponding to low reliability. Between the second and third collections (200$300 \mathrm{~m}$; 485-726 images), the ICC was 0.66 (moderate reliability; $95 \% \mathrm{Cl}, 0.14$ to 0.83 )

The pressure in the first metatarsal was similar between men and women (69.6 kPa vs $69.9 \mathrm{kPa} ; \mathrm{p}=0.58)$, right and left feet (69.8 kPa vs $69.7 \mathrm{kPa} ; \mathrm{p}=0.79$ ), dominant and non-dominant side (69.8 kPa vs $69.7 \mathrm{kPa} ; \mathrm{p}=0.93$ ) and physical or sedentary practitioners (70.6 kPavs 68.2 kPa; $p=0.07$ ). The pressure in the first metatarsal was similar between men and women (69.6 kPa vs $69.9 \mathrm{kPa}$; $\mathrm{p}=0.69)$, right and left feet (69.9 $\mathrm{kPa}$ vs $69.6 \mathrm{kPa} ; \mathrm{p}=0.82)$, dominant and non-dominant side (69.9 kPa vs $69.6 \mathrm{kPa} ; \mathrm{p}=0.30)$ and physical or sedentary practitioners (70.6 kPa vs $68.2 \mathrm{kPa}$; $\mathrm{p}=0.06$ ). The pressure in the calcaneus was similar between men and women (69.7 kPa vs $69.9 \mathrm{kPa} ; p=0.71)$, right and left feet (69.8 kPa vs $69.8 \mathrm{kPa} ; \mathrm{p}=0.82)$, dominant and non-dominant side $(69.8 \mathrm{kPa}$ vs $69.8 \mathrm{kPa} ; \mathrm{p}=0.28)$. However, the pressure in the calcaneus was $3.8 \%$ higher in individuals in physical activity practitioners $(70.7 \mathrm{kPa})$ compared to sedentary individuals (68.1 $\mathrm{kPa} ; \mathrm{p}=0.036)$. The plantar pressure was similar between men and women (69.8 kPa vs $69.9 \mathrm{kPa} ; \mathrm{p}=0.90)$, right and left feet (69.8 kPa vs $69.9 \mathrm{kPa} ; \mathrm{p}=0.88$ ), dominant and non-dominant side (69.8 kPa vs $69.9 \mathrm{kPa} ; \mathrm{p}=0.24)$. However, total plantar pressure was $3.8 \%$ higher among physical activity practitioners $(70.8 \mathrm{kPa})$ compared to sedentary individuals (68.2 $\mathrm{kPa} ; \mathrm{p}=0.04)$.

\section{DISCUSSION}

Currently, baropodometry is an important method to assist clinical practice and research in the diseases of the locomotor system. ${ }^{1}$ One of the main problems of baropodometry is the low reproducibility and interference of factors that can generate bias, such as sensor technology, spatial system resolution, pressure distribution analysis and calibration procedures. ${ }^{11}$ This study evaluated a insole baropodometry system with wireless technology whose results showed that the system is sensitive to evaluate the plantar pressure of normal individuals, with moderate reliability after the initial adaptation session.
A moderate agreement was identified for the insole system developed to measure plantar pressure. When singly considering the first gait cycle in our analysis, i.e., the evaluation of the pressure values generated in the first $100 \mathrm{~m}$ (242 images), the agreement assessment was low, probably because it refers to the adaptive cycle to the insole. In the final two cycles, after the adaptation period, we identified an improvement in the agreement and reliability of the measurements. Among the studies performing baropodometers of the insole type, possible difficulties in use and therapeutic application were suggested, in addition to discrepancy of pressure values when compared to other methods of analysis. ${ }^{2,11}$ In insoles, exists an adaptation inside the shoes, so it may undergo variations in the pressure distribution secondary to the shape of the base of the footwear, sole and hardness of the external materials. ${ }^{2}$ Our results corroborate the findings of Melvin et al., ${ }^{12}$ who suggested that from 166 steps there is accommodation to new footwear and exists an acclimatization distance for dynamic evaluation with insoles.

We identified relative consistency in plantar pressure values in anatomical areas where higher pressure concentrations usually occur. This consistency can be explained by choosing the moment of total contact of the foot with the insole, which represents the medium support phase of the gait in which, theoretically, has regular distribution of pressure along the plantar surface of the foot. Additionally, we applied as inclusion criteria only volunteers with asymptomatic feet, without deformities, metatarsalgia or callosities, which selects individuals with more uniform distribution potential of the pressure on the sole of the foot. The comparison of gait parameters evaluated by platform and insole system, and between insole instrument and platform, showed significantly lower mean values of the peak pressure of the metatarsophalangeal region in insole instruments, using footwear, when compared with the values generated by the platform. ${ }^{13}$ This could occur by increasing the contact area inside the shoe, created by the contour of the insole. ${ }^{14}$ We did not identify differences in pressure between genders, side or dominance. However, we identified that individuals who practice physical activity have plantar pressure in the heel and whole foot about $4 \%$ higher compared to sedentary individuals. This result compares the outcome obtained by Feka et al., ${ }^{15}$ who evaluated the effect of sports practice on static baropodometry. When analyzing 173 women, no significant differences were identified in the areas of plantar surfaces evaluated between the groups of athletes and sedentary. ${ }^{15}$ These findings can be explained by the methodology performed, which evaluated a static moment of total contact of the foot with the soil, in which the distribution is considered more uniform. Conversely, existents studies suggests differences in plantar pressure between the variables analyzed, and future studies need to include dynamic evaluation at different times of the gait cycle in order to identify possible pressure differences. ${ }^{15}$

Our study has limitations that must be emphasized. First, we included a small sample of healthy individuals, in which we expected to be no significant hyperpressure points due to the absence of deformities in the feet. Thus, the reliability obtained cannot be extrapolated to pathological situations. Second, variations in the size of the feet may have caused measurement bias in relation to the surface of the insole, which had a unique size for all individuals. Third, we did not evaluate the individuals in platform baropodometry concomitantly to compare the pressure values and accuracy of the insole system. Fourth, a study of kinetics of simultaneous gait was not performed using the insole, making it possible to determine exactly which gait phase corresponded to a certain pressure value. We tried to compensate this limitation by analyzing moments of total contact of the foot in the insole, which theoretically would be equivalent to the medium support in the gait cycle. Fifth, although we evaluated, in theory, areas of higher plantar pressure corresponding to the 
head of the first and fifth metatarsals and the heel, the areas were visually selected subjectively by the evaluator. It is worth noting that this insole coupled to a low-cost wireless system is an instrument that is easy to use, lightweight, mobile, capable of recording natural gait in a practical and safe way and with value of correspondence with the power platforms and presenting moderate reliability after the adaptation period.

\section{CONCLUSION}

We identified that the flexible and wireless insole baropodometry system was able to evaluate plantar pressure in healthy individuals with moderate reliability from the adaptation period. Future studies are important to establish the clinical applicability and accuracy of the system in physical therapy evaluations and in surgical practice.

AUTHORS' CONTRIBUTIONS: Each author contributed individually and significantly to the development of this article. LBO: search of articles, data analysis and writing of the article, study design and approval of the final article; DAM: study design, data analysis and approval of the final article; ACJ: study design data analysis and approval of the final article; MCMD: study design, data analysis and approval of the final article; RGP: study design, data analysis and approval of the final article.

\section{REFERENCES}

1. Ramanathan AK, Kiran P, Arnold GP, Wang W, Abboud RJ. Repeatability of the Pedar-X 1 in-shoe pressure measuring system. Foot Ankle Surg. 2010;16:70-3.

2. Saito M, Nakajima K, Takano C, Ohta Y, Sugimoto C, Ezoe R, et al. An in-shoe device to measure plantar pressure during daily human activity. Med Eng Phys. 2011;33(5):638-45.

3. Varoto R, Oliveira GC, Lima AVF, Critter MM, Cliquet A Jr. A low cost wireless system to monitor plantar pressure using insole sensor: feasibility approach. Proceedings of the 10th International Joint Conference on Biomedical Engineering Systems and Technologies; 2017 Feb 21-23; Porto, Portugal. New York: Springer; 2018. p. 207-14.

4. Giacomozzi C. Appropriateness of plantar pressure measurement devices: a comparative technical assessment. Gait Posture. 2010;32(1):141-4.

5. Hurkmans HLP, Bussmann JBJ, Benda E, Verhaar JAN, Stam HJ. Techniques for measuring weight bearing during standing and walking. Clin Biomech (Bristol, Avon). 2003;18(7):576-89.

6. Randolph AL, Nelson M, Akkapeddi S, Levin A, Alexandrescu R. Reliability of measurements of pressures applied on the foot during walking by a computerized insole sensor system. Arch Phys Med Rehabil. 2000;81(5):573-8.
7. Chen S, Lach J, Lo B, Yang GZ. Toward pervasive gait analysis with wearable sensors: a systematic review. IEEE J Biomed Health Inform. 2016;20(6):1521-37.

8. Hughes J. The clinical use of pedobarography. Acta Orthop Belg. 1993;59(1):10-6.

9. Koo TK, Li MY. A guideline of selecting and reporting intraclass correlation coefficients for reliability research. J Chiropr Med. 2016;15(2):155-63.

10. Portney, LG, Watkins MP. Foundations of clinical research: applications to practice. 3rd ed. New Jersey: F. A. Davis Company; 2015.

11. Baumfeld D, Baumfeld T, Rocha RL, Macedo B, Raduan F, Zambelli R, et al. Reliability of baropodometry on the evaluation of plantar load distribution: a transversal study. Biomed Res Int. 2017;2017:5925137.

12. Melvin JMA, Preece S, Nester CJ, Howard D. An investigation into plantar pressure measurement protocols for footwear research. Gait Posture. 2014;40(4):682-7.

13. Chevalier TL, Hodgins $\mathrm{H}$, Chockalingam N. Plantar pressure measurements using an in-shoe system and a pressure platform: a comparison. Gait Posture. 2010;31(3):397-9.

14. Cavanagh PR, Hewitt FG Jr, Perry JE. In-shoe plantar pressure measurement: a review. Foot. 1992;2(4):185-94.

15. Feka K, Pomara F, Russo G, Piccione MC, Petrucci M, Giustino V, et al. How do sports affect static baropodometry? An observational study among women living in southern Italy. Hum Mov. 2019;20(1):9-16. 